\title{
Tensor Arithmetic, Geometric and Mathematic Principles of Fluid Mechanics in Implementation of Direct Computational Experi- ments
}

\author{
Alexander Bogdanov ${ }^{1, a}$ and Vasily Khramushin ${ }^{1, b}$ \\ ${ }^{1}$ St. Petersburg State University, Universitetskii prospect 35, 198504 St.Petersburg, Russia
}

\begin{abstract}
The architecture of a digital computing system determines the technical foundation of a unified mathematical language for exact arithmetic-logical description of phenomena and laws of continuum mechanics for applications in fluid mechanics and theoretical physics. The deep parallelization of the computing processes results in functional programming at a new technological level, providing traceability of the computing processes with automatic application of multiscale hybrid circuits and adaptive mathematical models for the true reproduction of the fundamental laws of physics and continuum mechanics.
\end{abstract}

\section{Introduction}

The history of the Computational Fluid Dynamics (CFD) evolved in the vision rapid growth of computing resources availability and periodic assessments of how much tera- or exa-flops might be sufficient to solve all the problems of this science using pretty archaic mathematical methods. That said, attempts of Russian scientists [1,2] to go back to the formulation of the computational problem based upon the synthesis of initial physical principles and to approach computational experiments projecting from new positions and to go towards direct simulation of flows looked extremely dissonant. However, in-depth analysis of the purely physical problems in mathematical models creation and applied algorithms design in CFD was made as long ago as back in 1974 [3].

The first alarming signals sounded at the beginning of the century, when it turned out that most of the standard programs, if portable to servers with multicore processors at all, scale very poorly to say the least. At present, when most of computing complexes are heterogeneous, no ideas for the transfer of standard CFD programs onto them are noticeable. And the reason for this is clear the problem of the dynamics of elementary volume and geometric aspects of the dynamics are related so strongly, that the amount of diverse information that must be exchanged at each step of the calculation is comparable to the sheer volume of the data processed. And none of the hybrid processor is capable to cope with that. By now, it has become apparent that formal parallelism using vectorization of processing threads is lacking as there's a need for total control and hybrid fine-tuning of computation processes depending upon the physical condition of the liquids simulated. The development of continuous-corpuscular methods based on the "Large particles method" [2], leads to

\footnotetext{
ae-mail: a.v.bogdanov@spbu.ru

be-mail: v.khram@gmail.com
} 
computational models of tensor mathematics with independent status control of each computational cell-liquid particle, for which the computational algorithms as well as functional logic of physical phenomena and processes synthesis is being provided by arithmetic logic cores operating in parallel, that exactly matches the trends in the development of computer technology at the requests for the graphic visualization of three-dimensional spatial phenomena and dynamic processes with them. It is the use of tensor algebra for the direct simulation of physical phenomena and processes as part of the generalized tensor mathematics that allows to effectively synthesize hydrodynamic and geometrical aspects of the computing process in general. It's long been understood in field theory. Such a program for quantum gravity was outlined in [4], and then brilliantly realized in [5]. CFD problems have the very same nature, thus it is natural here to utilize the approach based on tensor mathematics for them too. And creation of algorithms for work with tensor numeric objects on modern hybrid systems gets realized in a natural way.

\section{Mathematical Formalization of Continual-Corpuscular Simulation in Fluid Mechanics}

Tensor mathematics is focused on the creation of direct computational experiments in solving practical problems of fluid mechanics. The continual-corpuscular approach is based on the numerical scheme of the first order with a consistent difference in the integration of the laws of motion of conjugate phases of scalar argument, i.e., time. Division of the computing stages by the physical processes enables continuous monitoring and hybrid evolution of mathematical relationships according to assessment of the current state of the simulated continuous medium, taking into account the intensity of the physical interaction between adjacent corpuscles as virtual numeric objects. The canonical representation of the laws of fluid mechanics allows strictly and unambiguously to associate numerical objects with arithmetic and logical operations and complex geometric algorithms, including the use of fast interpolation for unregularized grid spaces.

The consequent spatial integration of the first order involves grid and corpuscular approaches, formalized by means of the inertial mass tensor: $\mathbf{m}=m_{k}^{i}[\mathrm{~kg}]$ - which is used as a numeric object to fix pre-history, i.e., inertia in motion and local deformation of the simulated liquid particles that formed an algorithmic sequence of balanced prediction and correction of motion by a curved path for the fluid particles from the dynamically changing internal energy.

External mass force in the absolute coordinate system: $\vec{f}\left[\mathrm{~kg} \mathrm{~m} / \mathrm{s}^{2}\right]-$ in vector interpretation of the second Newton law is represented by the derivative of the momentum: $\vec{v} \cdot \mathbf{m} / t-$ in time (Fig. 1):

$$
\vec{f} \cdot t=\vec{v} \cdot \overrightarrow{\mathbf{m}}
$$

At the stage of continuous matter of computational experiment the vector fields are specified by the absolute velocity of the free movement of fluid particles $\vec{V}[\mathrm{~m} / \mathrm{s}]-$ and the local velocity $\vec{v}[\mathrm{~m} / \mathrm{s}]-$ under the influence of external mass forces $\vec{f}\left[\mathrm{~kg} \mathrm{~m} / \mathrm{s}^{2}\right]$ with the internal energy and inertia of $\overrightarrow{\mathbf{m}}=\boldsymbol{\vee} \cdot \hat{\mathbf{r}}$ $[\mathrm{kg}]$ - of specific particle: $\hat{\mathbf{r}}\left[\mathrm{m}^{3}\right]$ - in the product with tensor $\stackrel{\vee}{\rho}\left[\mathrm{kg} / \mathrm{m}^{3}\right]$, which formalizes the prehistory actions of volume force of inertia and of surface complex of living forces - the inherent energy of a liquid corpuscle. The center of mass and any inclusions inside a corpuscle $\overleftarrow{a}\left[\mathrm{~m}^{-2}\right]-$ move in time $t$ [s] in dependence on the environmental conditions $\stackrel{<}{\mathbf{m}}\left[\mathrm{kg}^{-1}\right]-$ in the vicinity of the nodes of the computational grid $\vec{\Omega}_{\Omega}[\mathrm{m}]$ - inside the spatial cells $\mathbf{r}\left[\mathrm{m}^{-3}\right]$ :

$$
+\vec{A}=\vec{R}+\vec{V} \cdot t+\vec{f} /\left(2 \mathbf{\mathbf { m }} \cdot t^{2}\right)+\overleftarrow{a} \cdot\left(\hat{\mathbf{r}}+\hat{\mathbf{v}} \cdot t+\hat{\mathbf{f}} /\left(2 \mathbf{\mathbf { m }} \cdot t^{2}\right)\right)
$$


where ${ }^{+} \vec{A}[\mathrm{~m}]$ is the point location at new (next) time step; $\vec{R}[\mathrm{~m}]$ is the original location of numerical cell center; $\hat{\mathbf{f}}\left[\mathrm{N} \mathrm{m}^{2}\right]$ is the surface tensions tensor dynamically approximated by adjacent cells and boundary conditions.

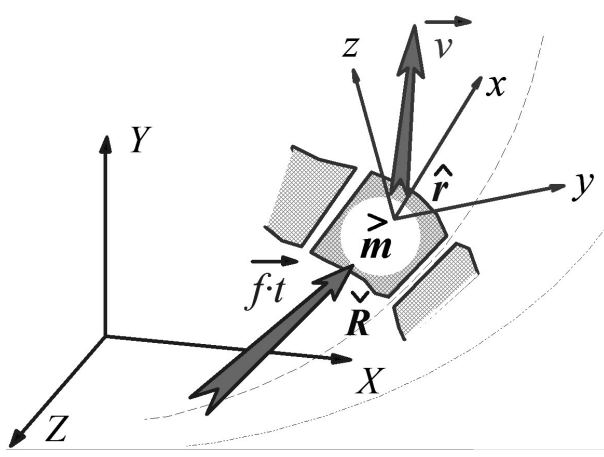

Figure 1. Separation of the calculating stages by the physical processes includes tensor mass as a geometric operator which corrects the motion of the fluid particles by the Vis viva (inner energy) and inertia at the conjugate stages of simulations in the time

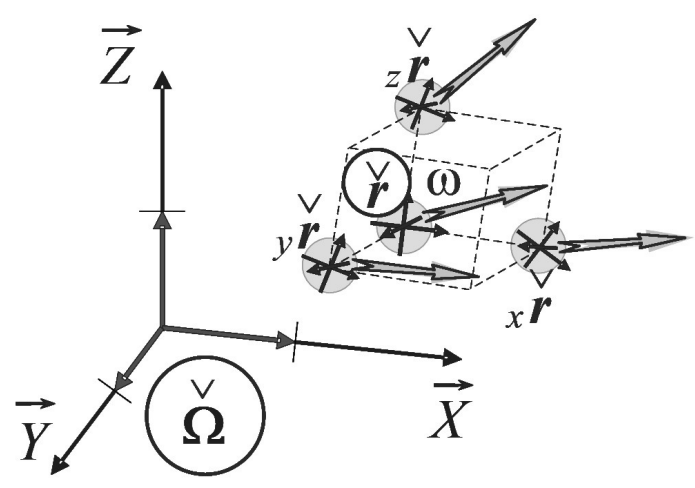

Figure 2. At the corpuscular stage of computational experiment an estimate of the current rheological state of the continuum in the initial nodes of the computational grid is made

The conjugate corpuscular stage of computations generates an updated kinematic field of local velocities' trivectors $\hat{\mathbf{v}}\left[\mathrm{m}^{3} / \mathrm{s}\right]$ - specifying the deformation displacements relative to shifting corpuscles: $\hat{\boldsymbol{\varepsilon}}\left[\mathrm{m}^{3}\right]$ - for reverse interpolation - secondary difference integration (Fig. 2) with fixing the tensions $\hat{\sigma}\left[\mathrm{kg} \mathrm{m}^{2} / \mathrm{s}^{2}\right]-$ in the immediate vicinity of the virtual numeric objects:

$$
\stackrel{<}{\boldsymbol{\sigma}}=(\hat{\boldsymbol{\varepsilon}} \cdot \stackrel{\mathrm{c}}{\mathbf{c}}+\hat{\mathbf{v}} \cdot \hat{\boldsymbol{\eta}}) \times \lambda \quad[\mathrm{N} \cdot \mathrm{m}]
$$

where $\stackrel{\vee}{\mathbf{c}}\left[\mathrm{N} / \mathrm{m}^{3}\right]$ - tensor modulus of elastic deformation; $\hat{\boldsymbol{\eta}}\left[\mathrm{N} \mathrm{s} / \mathrm{m}^{3}\right]$ - tensor of viscosity coefficients; $\lambda$ $[\mathrm{m}]$ - the scale factor, which characterizes the distance between the interacting virtual energy objects - the large particles of liquid.

Note that the tensors of tensions and local velocities may be degenerate. Change of basic sets without possibility of their inversion is performed only in multiplication operations with tensors of the form $\hat{\mathbf{v}} \cdot \stackrel{\vee}{\mathbf{r}}=\mathbf{v}\left[\mathrm{s}^{-1}\right]$ - or by algorithmic synthesis on the base of interpolation estimates of the environmental fluid state.

By analogy [6] inside the moving liquid particles a scalar function of the temperature distribution is involved, that is spread between the free particles by negative gradients; or change in the density of the elastic gas related to velocity of sound as function of the divergence of velocity and pressure values, including refinements imposed by the Bernoulli law and with the consideration of internal thermal energy, which altogether synthesizes the extended solution of applied aero- and hydromechanics problems.

As the main loop in the computer experiment, the sequence of algorithms intended for matching kinematic and rheological characteristics of the physical field in explicit numerical schemes, reducible to the mode of sequential setting of non-stationary computational processes arising from mathematical models for viscous, elastic and compressible fluids is accepted. 


\section{Tensor Representation of the Basic Laws of Fluid Mechanics}

The formal construction of physical objects and operations in the tensor mathematics [7] leads to strict definitions for the kind of "model of world" of computational fluid mechanics: 1) continuous corpuscular computing model of the method of "large particles" [2] in the tensor recording is reduced to a double linear difference interpolation of the physical fields (instead of integrating the equations of motion of the second order); 2) the movement and interaction of large liquid particles is built in terms of the operations of the product that more precisely corresponds to the physics of spatial processes (there are no restrictions on the smallness of the differential approximation); 3) the use of explicit numerical schemes and of discrete numeric fields serves to increase the effectiveness of direct computational experiments, and do not exclude the possibility of monitoring the correctness and, if necessary, involving of hybrid schemes to achieve adequate engineering results of direct numerical simulation.

The law of motion for a particle continuum:

$$
\vec{F}=\vec{W} \cdot \overrightarrow{\mathbf{m}}=\vec{W} \cdot \hat{\boldsymbol{\rho}} \cdot \stackrel{\vee}{\mathbf{r}}
$$

The Newton's viscous stress tensor:

$$
{\stackrel{<}{\mathbf{f}_{N}}}=\hat{\mathbf{v}}_{N} \cdot \stackrel{\vee}{\boldsymbol{\eta}} / \lambda=\overrightarrow{\mathbf{v}}_{N} \cdot \overrightarrow{\boldsymbol{\eta}} / \lambda ; \quad[\mathrm{N} / \mathrm{m}] .
$$

The Hook's elastic stress tensor:

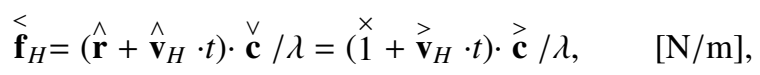

wherein the tensor of local velocities $\hat{v}={ }_{+} \vec{V}_{i}-{ }_{0} \vec{V}_{i}$ (Fig. 2); rheological tensors $\mathbf{m}=\stackrel{v}{\boldsymbol{\rho}} \cdot \hat{\mathbf{r}}[\mathrm{kg}]-$ tensor of mass inertia; $\hat{\mathbf{r}}\left[\mathrm{m}^{3}\right]$ - tensor of form; $\stackrel{\vee}{\rho}\left[\mathrm{kg} / \mathrm{m}^{3}\right]$ - "conditional density" of saving the pre-history of deviations - internal Vis viva (local forces) of moving corpuscles; $\overrightarrow{\boldsymbol{\eta}}[\mathrm{kg} / \mathrm{s}]$ - tensors of dynamic viscosity and stiffness $\overrightarrow{\mathbf{c}}[\mathrm{kg}]$ of real fluid, $\lambda$ - short distance interaction of adjacent particles.

The computational model comprises rheological properties of the fluid: viscosity and elasticity, while the ratio of the intensities of the resulting tensions determines the appearance of the critical flow regimes with the formation of jets, vortex layers and cavitations breaks. Under the influence of the internal stress tensor, a fluid particle gets velocity increment to internal (closed) movement which is traditionally represented by three dynamic states:

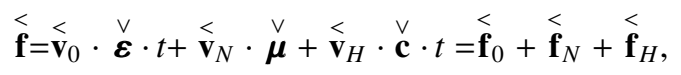

where the tensor ${\stackrel{<}{\mathbf{f}_{0}}}_{0}$ is the pressure, $\stackrel{\vee}{\boldsymbol{\varepsilon}}$ is the compression ratio. The dynamic coefficients $\stackrel{\vee}{\boldsymbol{\mu}}, \stackrel{\vee}{\mathbf{c}}, \boldsymbol{\vee} \boldsymbol{\varepsilon}$ differ from the kinematic ones by elimination of the scalar density value $\rho$. The resulting characteristic polynomial is used to assess the state of the liquid where the rheological parameters appear as the main invariants of the tensor of the convective velocities:

$\mathbf{v}_{0}: \mathbf{I} \neq 0$ - compressibility $\rightarrow$ cavitation density gap;

$\dot{\mathbf{v}}_{N}: \mathbf{I I} \neq 0$ - turn $\rightarrow$ formation of free jet or turbulent vortex;

$\dot{\mathbf{v}}_{H}:$ III $\neq 0$ - deformation, net $\rightarrow$ if the other invariants of the tensors are zero.

For example, if the computed cell with an attached vortex makes the determinant of internal field of convective velocities equal to zero in a conjugated point, i.e., in the point at the center of mass of free conjugate liquid particle, that will determine the emergence of a free turbulent vortex inside a large liquid particle. Such hybrid algorithm can be used either outside the approximated resolution for relatively coarse grids, or to improve decisions when the grid area in the zone of vortex shedding should condense. 


\section{On Peculiarities of the Tensor Generalization of Applied Fluid Mechanics Problems}

At stages of computing experiment the velocity field $\hat{\omega}\left[\mathrm{m}^{3} / \mathrm{s}\right]$ is formalized with a potentially degenerate tensor that satisfies the traditional additive construction of the flow model ${ }^{+} \hat{\mathbf{r}}={ }^{0} \hat{\mathbf{r}}+\hat{\mathbf{v}} \cdot t\left[\mathrm{~m}^{3}\right]-$ in absolute time: $t$ [s]. The definition of the tensor - affinor and expansion of Cauchy-Helmholtz [8] for spatial displacement is true: expansion (divergence); twist (rotor) and the deformation (shear).

Discrimination of the ball (diagonal) tensor $\stackrel{<}{\mathbf{v}_{0}}$ - such that the trace of the deviatory residue: $\operatorname{tr} \stackrel{\mathbf{v}}{*}^{*}$ - would be equal to zero [9]:

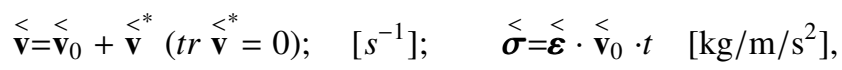

where tensor determinant $\stackrel{<}{\sigma}\left[\mathrm{kg} / \mathrm{m} / \mathrm{s}^{2}=\mathrm{N} / \mathrm{m}^{2}\right]$ - determines the pressure, $\stackrel{<}{\boldsymbol{\varepsilon}}$ - volume compression ratio - Young's modulus.

Symmetrization of deviatory tensor $\mathbf{v}^{*}$ - sets the shear stress $\stackrel{\boldsymbol{\tau}}{H}_{H}\left[\mathrm{~N} / \mathrm{m}^{2}\right]-$ according to Hooke's law within the large particle continuum:

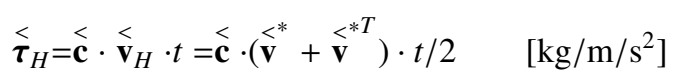

with the elastic modulus $\stackrel{<}{\mathbf{c}}\left[\mathrm{N} / \mathrm{m}^{2}\right]$ and symmetric tensor with respect to local displacements within a large fluid particle $\hat{\mathbf{v}}_{H} \cdot t$.

Skew-symmetric superposition with the components of the deviatory tensor separates the initial impulse to the rotation of the liquid particles, with the emergence of stress ${\stackrel{<}{\tau_{N}}}_{-}$in Newtonian definition of a viscous liquid mechanics:

$$
\stackrel{<}{\boldsymbol{\tau}}_{N}=\grave{\boldsymbol{\eta}} \cdot \dot{\mathbf{v}}_{N}=\stackrel{<}{\eta} \cdot\left(\stackrel{<}{\mathbf{v}}^{*}+\stackrel{<}{\mathbf{v}}^{* T}\right) / 2
$$

with the viscosity coefficient matrix $\stackrel{\iota}{\eta}\left[\mathrm{N} \mathrm{s} / \mathrm{m}^{2}\right]$ - in a product with the tensor of local deformations velocities $\stackrel{\mathbf{v}}{N}_{N}\left[\mathrm{~s}^{-1}\right]$.

The rheological characteristics of the liquid state $\stackrel{<}{\boldsymbol{\varepsilon}}\left[\mathrm{N} / \mathrm{m}^{2}\right], \stackrel{<}{\mathbf{c}}\left[\mathrm{N} / \mathrm{m}^{2}\right]$ and $\stackrel{<}{\boldsymbol{\eta}}\left[\mathrm{N} \mathrm{s} / \mathrm{m}^{2}\right]-$ can be defined by scalar coefficients in the traditional relationship between stress, local deformations and viscose movements of the continuous medium. In the tensor generalization the anisotropy of practical cross-linking components in the rheological characteristics of the liquid is implicitly taken into account by means of Poisson's coefficients.

Tensor mathematics completely covers the traditional models for linking internal stress with small displacements and strain velocities:

$$
\stackrel{<}{\boldsymbol{\varphi}}=\stackrel{<}{\boldsymbol{\sigma}}+\grave{\boldsymbol{\tau}}_{H}+\grave{\boldsymbol{\tau}}_{N}=\left(\stackrel{<}{\boldsymbol{\varepsilon}} \cdot \grave{\mathbf{v}}_{0}+\stackrel{<}{\mathbf{c}} \cdot \grave{\mathbf{v}}_{H}\right) \cdot t+\stackrel{<}{\boldsymbol{\eta}} \cdot \stackrel{\mathbf{v}}{N}_{N} \quad\left[\mathrm{~N} / \mathrm{m}^{2}\right]
$$

In order to bring the law of motion with inertial mass $\stackrel{<}{\mathbf{f}} \cdot t=\stackrel{<}{\mathbf{m}} \cdot \stackrel{<}{\mathbf{v}}[\mathrm{kg} / \mathrm{s}]-$ to the new relationships for the surface tension $\stackrel{<}{\varphi}\left[\mathrm{kg} / \mathrm{m} / \mathrm{s}^{2}\right]$, a factor with the dimension of the space vector is necessary, that corresponds by the physical meaning either to the vector of direction of movement for Zhukovsky theorem for the free vortex dipole, or to the distance between the interacting particles of the liquid. 


\section{Conclusion}

The geometrical construction of non-regularized grid spaces [10] in explicit numerical schemes of the first order for the sequential integration in conjugated stages in continuous-corpuscular computing experiments when modeling the transient processes in fluid mechanics is represented by sequential superposition upon the architecture of digital computers:

1. A continual corpuscular division of the stages of the computing experiment in fluid mechanics is constructed in the continuity space of physical values, initially defined in a single absolute coordinate system using scalar, vector and tensor characteristics of the state and the laws of transformation of the mobile and deformable continuous medium;

2. In multiplication operations with external (mass) and internal (surface) tensions for the computed time interval, the internal state of each computed cell is rebuilt in the similitude of the estimated independent large fluid particle accumulating internal energy and making influence on the kinematic reactions in the interpolation rebuilding of updated physical fields in the initial computational nodes;

3. In dealing with very complex physical laws of fluid mechanics, an additional third phase of calculations is introduced for monitoring and adjusting the internal state of the liquid particles, with the coordination of the laws of conservation of the mass and momentum of the free liquid particles from the point of alignment the calculations at the two main stages of the computational experiment for the scalar argument, the time.

\section{Acknowledgements}

The research was carried out using computational resources of Resource Center Computer Center of SaintPetersburg State University and supported by Russian Foundation for Basic Research (project N 13-07-00747) and St. Petersburg State University (projects N 9.38.674.2013, 0.37.155.2014).

\section{References}

[1] Dorodnitsin A.A., Selected works. V.1.2. (Moscow. Publication of the Computer Centre of the Academy of Sciences Russia 1997)

[2] Belotserkovsky O.M. and Davydov Yu.M., Method of large particles in the gas dynamics (Nauka, Moscow, 1982) 370 pp.

[3] Astarita G. and Marrucci G. Principles of non-Newtonian fluid mechanics (McGRAW-HILL Book Company, UK, 1974) 289 pp.

[4] DeWitt B.S., Dynamical theory of groups and fields (Gordon and Breach, N.-Y., 1965) 264 pp.

[5] Garrett Lisi A., An Exceptionally Simple Theory of Everything, arXiv:0711.0770v1 [hep-th] 6 Nov 2007

[6] Eglit M.E., The lectures on the basics of continuum mechanics (Publishing house of the Moscow University, Moscow, 2008) 318 pp.

[7] Khramushin V. N., Three-dimensional tensor mathematics of computational experiments in fluid mechanics. (FEB RAS, Vladivostok, 2005) 212 pp.

[8] Gotman A.Sh., The tensor calculus (Novosibirsk State Academy of Water Transport, Novosibirsk, 2007) 129 pp.

[9] Mac-Konnel A.J., Introduction to tensor analysis (Dover Publications, 2011) 352 pp.

[10] Wesseling P., Principles of Computational Fluid Dynamics (Springer Series in Computational Mathematics, 2001) $644 \mathrm{p}$. 